\title{
Subsecond Time Evolution of Type III Solar Radio Burst Sources at Fundamental and Harmonic Frequencies
}

\author{
Xingyao Chen ${ }^{1,2}$ (1), Eduard P. Kontar ${ }^{2}$ (1), Nicolina Chrysaphi ${ }^{2}$ (10), Natasha L. S. Jeffrey ${ }^{3}$ (10, Mykola Gordovskyy ${ }^{4}$ (1), \\ Yihua Yan $^{1,5}$, and Baolin $\operatorname{Tan}^{1,5}$ (10) \\ ${ }^{1}$ Key Laboratory of Solar Activity, National Astronomical Observatories, Chinese Academy of Sciences, Beijing 100101, People's Republic of China \\ ${ }^{2}$ School of Physics \& Astronomy, University of Glasgow, Glasgow G12 8QQ, UK \\ ${ }^{3}$ Department of Mathematics, Physics and Electrical Engineering, Northumbria University, Newcastle upon Tyne NE1 8ST, UK \\ ${ }^{4}$ School of Physics \& Astronomy, University of Manchester, Manchester M13 9PL, UK \\ ${ }^{5}$ School of Astronomy and Space Sciences, University of Chinese Academy of Sciences, Beijing 100049, People's Republic of China \\ Received 2020 April 10; revised 2020 October 16; accepted 2020 October 16; published 2020 December 10
}

\begin{abstract}
Recent developments in astronomical radio telescopes opened new opportunities in imaging and spectroscopy of solar radio bursts at subsecond timescales. Imaging in narrow frequency bands has revealed temporal variations in the positions and source sizes that do not fit into the standard picture of type III solar radio bursts, and require a better understanding of radio-wave transport. In this paper, we utilize 3D Monte Carlo ray-tracing simulations that account for the anisotropic density turbulence in the inhomogeneous solar corona to quantitatively explain the image dynamics at the fundamental (near plasma frequency) and harmonic (double) plasma emissions observed at $\sim 32 \mathrm{MHz}$. Comparing the simulations with observations, we find that anisotropic scattering from an instantaneous emission point source can account for the observed time profiles, centroid locations, and source sizes of the fundamental component of type III radio bursts (generated where $f_{\mathrm{pe}} \approx 32 \mathrm{MHz}$ ). The best agreement with observations is achieved when the ratio of the perpendicular to the parallel component of the wavevector of anisotropic density turbulence is around 0.25. Harmonic emission sources observed at the same frequency $\left(\sim 32 \mathrm{MHz}\right.$, but generated where $f_{\mathrm{pe}} \approx 16 \mathrm{MHz}$ ) have apparent sizes comparable to those produced by the fundamental emission, but demonstrate a much slower temporal evolution. The simulations of radio-wave propagation make it possible to quantitatively explain the variations of apparent source sizes and positions at subsecond timescales both for the fundamental and harmonic emissions, and can be used as a diagnostic tool for the plasma turbulence in the upper corona.
\end{abstract}

Unified Astronomy Thesaurus concepts: Solar radio emission (1522); Interplanetary turbulence (830); Radio bursts (1339); Solar radio flares (1342)

\section{Introduction}

Solar radio bursts are commonly considered to be a signature of acceleration and propagation of nonthermal electrons in the solar corona (e.g., Ginzburg \& Zhelezniakov 1958; Dulk 1985). In the standard type III solar radio burst scenario, nonthermal electrons propagate away from the Sun and generate Langmuir waves (e.g., Ginzburg \& Zhelezniakov 1958; Goldman 1983; Yoon et al. 2016), so that the radio emission is progressively produced at lower frequencies as the electrons responsible for radio emission propagate away from the Sun. The radio emission is produced at the fundamental and harmonic (twice the local plasma frequency) frequencies, so observations at the same frequency examine the harmonic emission from distances farther away from the Sun. At the same time, various propagation effects-including the refraction due to plasma density gradients and scattering by small-scale density fluctuations-significantly affect the apparent properties of the radio sources, including their time evolution, position, and size (Steinberg et al. 1971; Arzner \& Magun 1999; Kontar et al. 2017). Scattering in the inhomogeneous solar corona is also considered as a possible explanation for the decrease in the apparent brightness temperature of the quiet Sun at about

Original content from this work may be used under the terms of the Creative Commons Attribution 4.0 licence. Any further distribution of this work must maintain attribution to the author(s) and the title of the work, journal citation and DOI.
$30 \mathrm{MHz}$ (Aubier et al. 1971; Thejappa et al. 2007). Therefore, radio-wave scattering needs to be taken into account in the analyses of solar radio observations.

Scattering of radio waves in the solar corona has been extensively studied since the first solar radio observations (Fokker 1965; Steinberg et al. 1971; Steinberg 1972; Riddle 1974; Bougeret \& Steinberg 1977; Robinson 1983; Bastian 1994; Arzner \& Magun 1999; Thejappa et al. 2007; Ramesh et al. 2020). Consequently, Fokker (1965) performed the first numerical study of scattering on small-scale density inhomogeneities to estimate the apparent sizes and locations of solar radio sources. By accounting for absorption, irregular refraction by large-scale inhomogeneities, and isotropic scattering, Steinberg et al. (1971), Steinberg (1972), and Riddle (1974) extended the ray-tracing method to study the arrival time, intensity, and angular broadening of the scattered images. Arzner \& Magun (1999) considered the propagation of radio waves in an anisotropic, statistically inhomogeneous plasma using geometrical optics and the Hamilton equations, and successfully reproduced many features of radio bursts. Later, Thejappa et al. (2007) developed Monte Carlo simulations to study isotropic scattering effects focusing on the directivity of interplanetary type III bursts at frequencies ranging between 100 and $200 \mathrm{kHz}$. Krupar et al. (2018) simulated the scattering effects on low-frequency plasma radio emission and demonstrated that type III bursts can be used as a diagnostic tool for plasma density variations in the solar wind. 
Recent observations by Krupar et al. (2018) suggest that radio-wave scattering effects in the solar corona and interplanetary space dominate the observed duration of fundamental radio sources. Most of the radio-wave scattering simulations assumed that the density inhomogeneities are isotropic, but Kontar et al. (2019) found that the time duration and source size cannot be simultaneously reproduced using isotropic density fluctuations, and suggested that radio-wave scattering is strongly anisotropic.

Kontar et al. (2017) and Sharykin et al. (2018) observed the centroid positions, sizes, and areal extents of the fundamental and harmonic sources of a Type III-IIIb burst, and demonstrated that scattering effects dominate the observed spatial characteristics of radio burst images. Chrysaphi et al. (2018) illustrated that the spatial separation observed between the sources of split-band Type II bursts is consistent with radiowave scattering effects.

For the first time, in this study, Monte Carlo simulations of radio-wave propagation are used to investigate the time evolution, positions, and sizes of the apparent solar radio burst sources at subsecond scales. The simulations are performed both for the fundamental and harmonic components, with the aim of explaining the properties of type III-IIIb solar radio bursts observed by the LOw-Frequency ARray (LOFAR; van Haarlem et al. 2013).

The article is arranged as follows. Section 2 describes the anisotropic scattering and ray-tracing model. Section 3 describes the simulation results and their comparison with the radio burst observations. Section 4 presents the discussion and conclusions.

\section{Radio-wave Scattering Simulations}

\subsection{Equations}

Radio waves with angular frequency $\omega$ and wavevector $k$ in an unmagnetized plasma follow the dispersion relation $\omega^{2}=\omega_{\mathrm{pe}}^{2}+k^{2} c^{2}$, where $\omega_{\mathrm{pe}}=\sqrt{4 \pi e^{2} n(\boldsymbol{r}) / m_{e}}$ is the electron plasma frequency. Both the fundamental emission $\left(\omega \sim \omega_{\mathrm{pe}}\right)$ and second-harmonic radio waves $\left(\omega \sim 2 \omega_{\text {pe}}\right.$, produced farther away from the Sun) have their frequencies close to the local plasma frequency $\omega_{\text {pe }}$, and are hence sensitive to the electron density fluctuations via the plasma refractive index $\sqrt{1-\omega_{\mathrm{pe}}^{2} / \omega^{2}}$ (see, e.g., Pécseli 2012).

In this study we use the same numerical approach as in Kontar et al. (2019). The density fluctuations in the solar corona are assumed to be axially symmetric with respect to the local radial direction (the direction of the guiding magnetic field), so that the spectrum can be parameterized as a spheroid in wavevector space:

$$
S(\boldsymbol{q})=S\left(\left[q_{\perp}^{2}+\alpha^{-2} q_{\|}^{2}\right]^{1 / 2}\right),
$$

where $\alpha=h_{\perp} / h_{\|}$is the ratio of perpendicular and parallel correlation lengths, leading to the diffusion tensor components $D_{i j}$ :

$$
\begin{aligned}
& D_{i j}=\left[\frac{A_{i j}^{-2}}{\left(\boldsymbol{k} \mathrm{A}^{-2} \boldsymbol{k}\right)^{1 / 2}}-\frac{\left(\mathrm{A}^{-2} \boldsymbol{k}\right)_{i}\left(\mathrm{~A}^{-2} \boldsymbol{k}\right)_{j}}{\left(\boldsymbol{k} \mathrm{A}^{-2} \boldsymbol{k}\right)^{3 / 2}}\right] \\
& \times \frac{\omega_{p e}^{4}}{32 \pi \omega c^{2}} \alpha \int_{0}^{\infty} \widetilde{q}^{3} S(\widetilde{q}) d \widetilde{q} .
\end{aligned}
$$

Here $\mathbf{A}$ is the anisotropy matrix

$$
A=\left(\begin{array}{ccc}
1 & 0 & 0 \\
0 & 1 & 0 \\
0 & 0 & \alpha^{-1}
\end{array}\right)
$$

and $\widetilde{\boldsymbol{q}}=\mathrm{A} \boldsymbol{q}$ (so that $\boldsymbol{q}=\mathrm{A}^{-1} \widetilde{\boldsymbol{q}}$ ). The scattering tensor (Equation (2)) depends on the spectrum-averaged wavenumber of the density fluctuations

$$
\overline{q \epsilon^{2}}=\overline{q \delta n^{2}} \frac{1}{n^{2}}=\frac{1}{n^{2}} \int_{0}^{\infty} \widetilde{q}^{3} S(\widetilde{q}) \frac{4 \pi d \widetilde{q}}{(2 \pi)^{3}}
$$

and the anisotropy parameter $\alpha$. Therefore, knowing the mean wavenumber $\overline{q \epsilon^{2}}$, one can describe the scattering of radio waves in a turbulent solar plasma (Kontar et al. 2019).

In situ (Celnikier et al. 1983; Krupar et al. 2020) and remotesensing radio observations (Wohlmuth et al. 2001) suggest that the density fluctuations in the interplanetary space have an inverse power-law spectrum $S(q) \propto q^{-(p+2)}$, with the spectral index $p$ close to $5 / 3$. The power law is normally observed over a broad inertial range, from the outer scales $l_{0}=2 \pi / q_{0}$ to the inner scales $l_{i}=2 \pi / q_{i}$, so we consider

$$
S(q)= \begin{cases}0, & q>q_{0} \\ \text { const } \times \mathrm{q}^{-(p+2)}, & q_{i}<q<q_{0} . \\ 0, & q<q_{i}\end{cases}
$$

The parameters of the power-law spectrum with a Kolmogorov-like spectral index $(p=5 / 3)$ can be determined from ground-based or in situ observations (Tu \& Marsch 1993; Wohlmuth et al. 2001; Shaikh \& Zank 2010; Chen et al. 2018). Similar to Thejappa et al. (2007) and Krupar et al. (2018), we assume that the density fluctuations form a power-law spectrum with a spectral index of $p=5 / 3$.

The inner scale of the inhomogeneities depends on the heliocentric distance as $l_{i}=r /\left(6.955 \times 10^{5}\right)$ (Manoharan et al. 1987; Coles \& Harmon 1989) between $2-100 R_{\odot}$, and the outer scale varies as $l_{o}=0.23 \times r^{0.82}$ for distances $r$ from 4-80R $R_{\odot}$ (Wohlmuth et al. 2001). Hence, the mean wavenumber can be written as a function of heliocentric distance as follows:

$$
\overline{q \epsilon^{2}} \simeq 4 \pi l_{i}^{-1 / 3} l_{o}^{-2 / 3} \epsilon^{2}=C_{q} r^{-0.88},
$$

where $r$ is the distance from the solar center in units of solar radius $R_{\odot}, \epsilon^{2}=\left\langle\delta n^{2}\right\rangle / n^{2}$ is the variance of density fluctuations, and $C_{q}$ is a constant characterizing the level of density fluctuations in units of $1 / R_{\odot}$. Thus, $C_{q}$, which characterizes the density fluctuation properties, and $\alpha$, which characterizes their anisotropy, are the main input parameters in our numerical models. By the definition, $\epsilon$ is the integral over all wavenumbers, while the density fluctuations have a broad spectrum that over all wavenumbers is unknown. The $l_{o}$ and $l_{i}$, the outer and inner scales of the inhomogeneities, are also hard to estimate. Considering that the $\epsilon, l_{o}$, and $l_{i}$ are poorly known at the heliocentric distances of interest $\left(r=1.5-2 R_{\odot}\right)$, we will use $C_{q}$ as a free parameter to match the observations.

\subsection{Ray-tracing Simulation}

In our simulations, the radio emission source is assumed to be a point source at a heliocentric distance $r_{0}$. The source isotropically emits photons with a wavenumber $|\boldsymbol{k}|$, corresponding to the fundamental frequency $1.1 \omega_{p e}\left(r=r_{F}\right)$ or the 

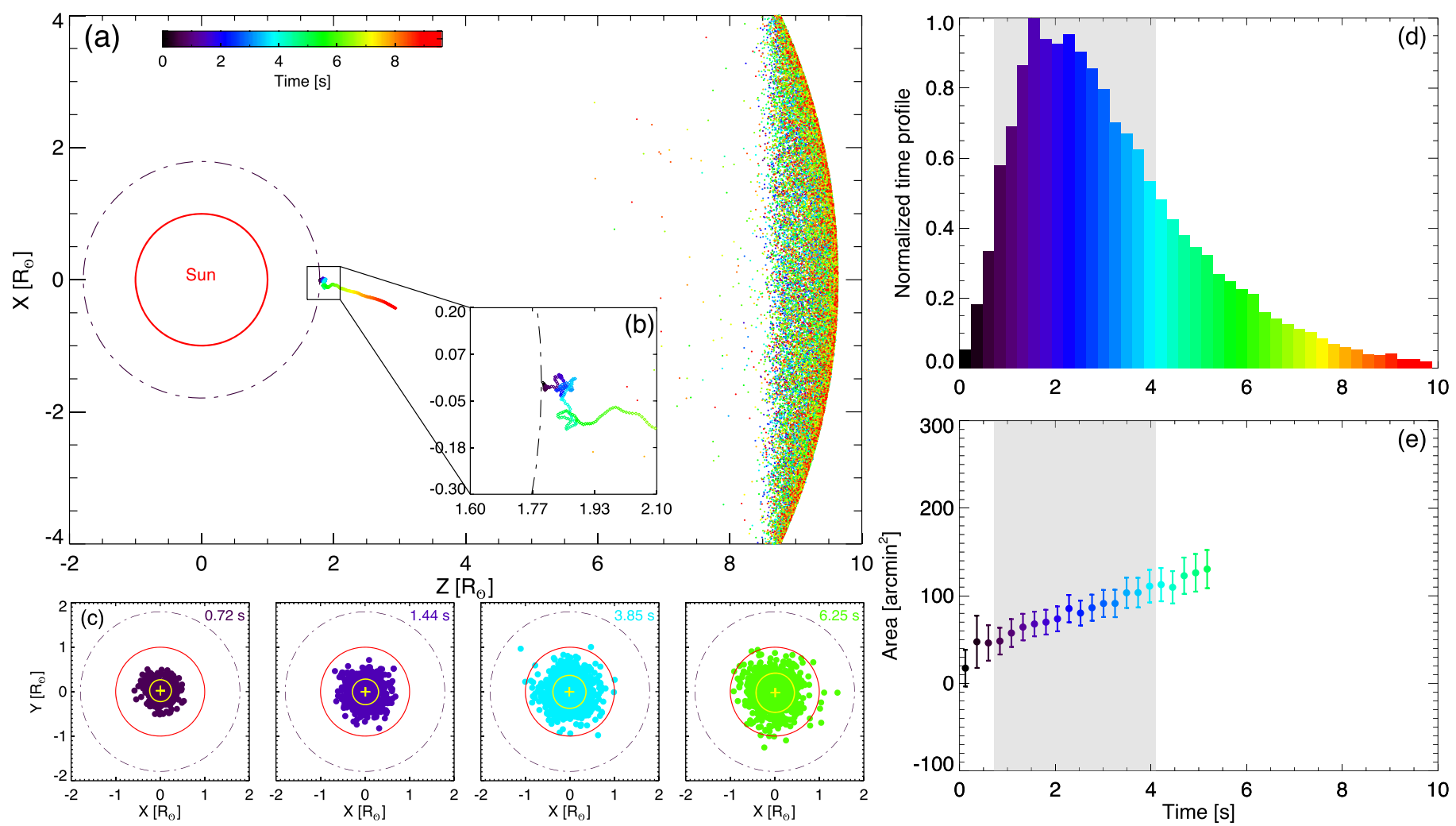

Figure 1. Schematic diagrams illustrating the ray-tracing simulations conducted assuming isotropic scattering $(\alpha=1)$ and turbulence levels given by $C_{q}=80 R_{\odot}^{-1}$, showing a small number of photons for clarity. The Sun is located at the center of the coordinate system with the $Z$-axis pointing toward the observer. The dasheddotted circle illustrates the distance corresponding to the initial source location $r_{0}$. The source is located at $\theta=0^{\circ}$. The different colors used represent different arrival times, where the free-space propagation time time has been subtracted. Panel (a) shows a single ray trajectory as a function of time after the photon's injection into the corona, and the photons arriving at distance $9.6 R_{\odot}$ (where scattering becomes negligible). Panel (b) is an enlargement of part of the single ray trajectory closer to the emission location. Panel (c) shows snapshot images for the different time moments. The yellow circle and plus sign represent the source's FWHM size and centroid location, respectively. The red circle represents the solar disk. Panel (d) shows a histogram of the photon arrival times. The gray area indicates the FWHM of the impulse. Panel (e) shows the FWHM size as a function of time. The error bars represent the one standard deviation uncertainty given by Equations (47) and (48) in Kontar et al. (2019).

harmonic frequency $2 \omega_{\mathrm{pe}}\left(r=r_{H}\right)$, where $\omega_{\mathrm{pe}}$ is the electron plasma frequency at the physical location of the source, i.e., at $r=r_{F}$ and $r=r_{H}$ for fundamental and harmonic frequencies, respectively. In this paper, we present simulations for emissions observed at $32.5 \mathrm{MHz}$, a frequency often imaged in LOFAR observations. The fundamental plasma emission of this frequency is generated at a heliocentric distance of $\sim 1.8 R_{\odot}$, while the harmonic is generated at $\sim 2.2 R_{\odot}$, according to the density model used (Equation (7)). The background density in the upper corona is assumed to be

$$
n(r)=4.8 \times 10^{9} r^{-14}+3 \times 10^{8} r^{-6}+1.39 \times 10^{6} r^{-2.3},
$$

which is an analytical approximation of the Parker density profile (Parker 1960), with $r$ expressed in solar radii.

In each numerical experiment, $2 \times 10^{5}$ rays are traced through the corona until all rays arrive at a sphere where the scattering is assumed to be negligible (i.e., $\omega$ pe $\ll \omega$ ). In our simulations, for a $32.5 \mathrm{MHz}$ source the radius of the "scattering corona" is set to be $r_{s} \sim 9.6 R_{\odot}$, where scattering becomes negligible, i.e., the total (photon path integrated) angular broadening between $r_{s}$ and the Earth is 0 .2. This allows us to calculate the typical observed angular size with a precision of $\sim 1 \%$ for typical type III burst sizes of $20^{\prime}$ at $32 \mathrm{MHz}$. Therefore, the largest uncertainty in the results is the statistical error due to the finite number of photons in the simulations, which is accounted for and illustrated throughout our analysis.

In addition to scattering, we also take into account the freefree absorption of radio waves according to Lifshitz \& Pitaevskii (1981). The observed brightness temperature is reduced by a factor of $e^{-\tau}$, where $\tau$ is the optical depth along the photon path in the simulations. The optical depth $\tau$ can be calculated as

$$
\tau=\sum_{i=0}^{N} \sqrt{\frac{2}{\pi}} \frac{e^{2} \ln \Lambda}{4 m_{e} c v_{T e}{ }^{3} \omega}\left(\frac{\omega_{\mathrm{pe}}{ }^{4}}{\sqrt{\omega^{2}-\omega_{\mathrm{pe}}^{2}}}\right) \Delta s_{i},
$$

where $v_{T e}=\sqrt{k_{B} T_{e} / m_{e}}$ is the electron thermal velocity, $\Lambda$ is the Coulomb logarithm ( $\sim 20$ in the solar corona), and $\Delta s_{i}$ denotes the distance interval in each time step (see Kontar et al. 2019 for details).

To illustrate the difference between anisotropic and isotropic ( $\alpha=1$ ) turbulence, we perform simulations for both. The simulation results for $\alpha=1$ are shown in Figure 1. All simulated photons (observed at $32 \mathrm{MHz}$ ) are initially located at $r_{0} \sim 1.8 R_{\odot}$ for the fundamental plasma emission and $r_{0} \sim$ $2.2 R_{\odot}$ for the harmonic plasma emission. Panel (a) shows the photon locations when they reach the boundary of the scattering corona, with different colors representing the time since the photon emission from the point source. The ray path of a randomly chosen photon is also illustrated (panel (b)). The 
radio waves are strongly scattered close to the source, making wave propagation diffusive (see, e.g., Bian et al. 2019). The scattering rate decreases with distance, and the refraction due to large-scale density inhomogeneities becomes more significant, causing some "focusing" of the radio waves.

In order to derive the apparent location and shape of the source, all rays arriving at the boundary of the "scattering corona" are projected to the plane of sky to create an image (in this case, the plane perpendicular to the line of sight and containing the center of the Sun). Photons with $0.85<k_{z} /$ $k<1$ (at the moment of leaving the "scattering corona") are used to create the source intensity map $I(x, y)$ similar to Jeffrey \& Kontar (2011). As done to observed emission images (see, e.g., Kontar et al. 2017), the simulated radio images were fitted with a 2D Gaussian in order to determine the centroid positions and the FWHM sizes, as shown in Figure 1(c).

The number of photons arriving at the "scattering corona" $\left(r_{s}=9.6 R_{\odot}\right)$ varies with time as shown in Figure $1(\mathrm{~d})$, where the depicted time is offset by the free-space light prorogation $\left(\left(r_{s}-r_{0}\right) / c\right)$, i.e., $t=t_{r_{s}}-\left(r_{s}-r_{0}\right) / c$. Thus, in the case of free-space photon propagation, all photons arrive at time $t=0$. However, due to the presence of density inhomogeneities giving rise to propagation effects, the observed pulse is broadened and the peak of the emission arrives later than freespace transport predicts. It should be noted that both the scattering and the large-scale refraction affect the photon propagation, but scattering produces the largest effect. Figure 1(d) demonstrates that for isotropic scattering with $C_{q}=80 R_{\odot}^{-1}$, the radio pulse peak will be delayed by about $2.5 \mathrm{~s}$ and the instantaneously injected photons will be observed as a $\sim 3.5 \mathrm{~s}$ long pulse. To show the dynamics of the radio source, images were simulated at different time moments, shown in Figure 1(c).

\section{Comparison with LOFAR Observations}

\subsection{Observations}

Solar radio type III bursts are believed to be generated by nonthermal electrons propagating along open magnetic field lines. The ultrahigh temporal $(\sim 10 \mathrm{~ms})$ and spectral $(12.5 \mathrm{kHz})$ resolutions of LOFAR (van Haarlem et al. 2013) allow us to observe the unique fine structures (striae) in type III bursts emitted between 30 and $80 \mathrm{MHz}$. The type III burst presented in this study was observed on 2015 April 16 at $\sim 11: 57$ UT (Kontar et al. 2017; Chen et al. 2018; Kolotkov et al. 2018; Sharykin et al. 2018). It is composed of hundreds of striae with a short duration $(\sim 1 \mathrm{~s})$ and a narrow bandwidth $(\sim 100 \mathrm{kHz})$ for both its fundamental and harmonic branches. Background density perturbations are believed to have strong effects on the the formation of the striae (Chen et al. 2018). Kontar et al. (2017) illustrated that the radio-wave scattering effects dominate the observed spatial characteristics of the radio sources. To obtain a better understanding of the propagation of radio waves and the density inhomogeneity in the corona, we compare our radio-wave propagation simulations with the observed properties of the striated type III burst.

\subsection{Fundamental Emission}

Simulations of emissions at $32.5 \mathrm{MHz}$ reveal the time evolution, apparent motions, and sizes of radio sources, which can be directly compared with the type III-IIIb LOFAR observations. To match the observations, we consider three parameters: the level of density fluctuations $C_{q}$, anisotropy $\alpha$, and the heliocentric angle $\theta$ of the source.

Similar to the observations, the temporal evolution of the sources at a given frequency is characterized in the decay phase of the temporal profile. We then apply exponential fits to the decay phase and describe the decay times as the half-width at half-maximum (HWHM) from the fits (similar to, e.g., Reid \& Kontar 2018; Krupar et al. 2020). The one standard deviation uncertainty calculated during fitting is used as the error in the measurements of decay time.

Figure 2 compares the main observed characteristics of type III sources with those produced by simulations for $\alpha=1$ (isotropic scattering) and $C_{q}=80 R_{\odot}^{-1}$. The gray shaded area in all panels indicates the decay time derived from the observations. The top panels show the time profiles of the observed source (red curve) and of the simulated source with respect to the heliocentric angle. It can be seen that the FWHM of the impulse produced by the instantaneous source is broadened to about $3.5 \mathrm{~s}$, which is substantially longer than the FWHM of the observed impulse (about 1.1 s). Similarly, the decay time in the simulations is approximately $2.5 \mathrm{~s}$ long, while the observed decay time is only $\sim 0.5 \mathrm{~s}$.

The relative centroid positions were represented by the offset from the peak in order to better compare with Figure 4(b) from Kontar et al. (2017). From Figure 2(b), the simulated source also demonstrates a much slower apparent motion compared to the observed source. During the decay phase, the observed source moves by approximately $65^{\prime \prime}$ (in $\sim 0.5 \mathrm{~s}$ ), while the simulated source-during the same period $(\sim 0.5 \mathrm{~s})$ - moves by less than $5^{\prime \prime}$, and moves only by $12^{\prime \prime}$ during the entirety of the simulated decay phase $(\sim 2.5 \mathrm{~s})$. Evidently, the source positions do not change substantially in the case of isotropic scattering, which is inconsistent with the LOFAR observations shown by the red line in Figure 2.

The observed source areas, which were corrected for the FWHM area of the LOFAR beams $\left(\sim 110 \operatorname{arcmin}^{2}\right.$ at $32.5 \mathrm{MHz}$ ), vary from $\sim 300$ to $\sim 440 \mathrm{arcmin}^{2}$ during the decay phase ( $\sim 0.5 \mathrm{~s}$ for the fundamental emissions). From the isotropic scattering simulations, the simulated size for the fundamental emission ranged from $\sim 60$ to $\sim 100 \mathrm{arcmin}^{2}$ during the $2.5 \mathrm{~s}$ of the decay phase, which is substantially different from the LOFAR observations.

Although the simulated decay time was longer than the observed, the apparent source size was 4 times smaller. Therefore, under the isotropic scattering assumption, the simulated decay time and source size cannot both agree with the observations, irrespective of how weak or strong the scattering effects are set to be, given that stronger scattering will result in both larger source sizes and longer decay times. Furthermore, in the case of isotropic scattering, the simulated apparent motion of centroids was much smaller than that observed. Therefore, it is clear that isotropic scattering cannot simultaneously explain the size and temporal evolution of a typical type III radio source (as illustrated by Kontar et al. 2019) and should not be used for simulations of radio-wave transport.

\subsection{Anisotropic Scattering}

Given that the isotropic description of scattering was shown to be insufficient, we simulate anisotropic scattering with anisotropy values $\alpha$ ranging from 0.2 to 0.3 , so that the perpendicular density fluctuations have a stronger effect on the 

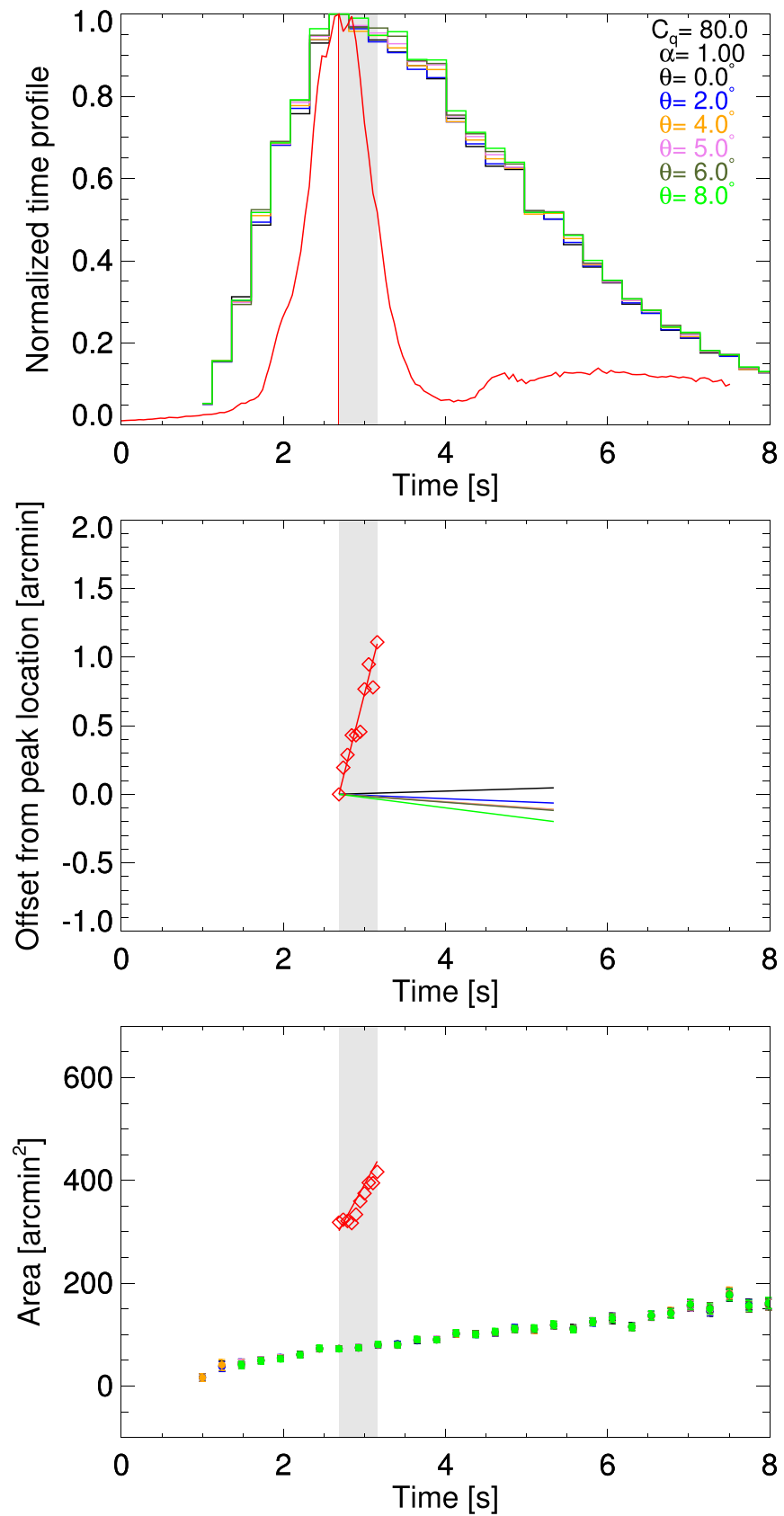

Figure 2. Simulations of fundamental plasma emission from an instantaneous point source scattered by isotropic turbulence. The top panel shows the time profiles, the middle panel shows the centroid shifts, and the bottom panel shows the apparent source sizes with respect to the time after free-space propagation time. The frequency of the simulated source was taken to be 32.5 $\mathrm{MHz}, C_{q}=80 R_{\odot}^{-1}$, and the heliocentric angles $\theta$ of the source varied from $0^{\circ}$ to $8^{\circ}$, represented by the different colors, as indicated in the legend of the top panel. The red data represent LOFAR observations aligned to match the emission peak from Kontar et al. (2017). The gray shaded area represents the decay time of the burst observed by LOFAR.

radio-wave propagation than the respective parallel component (see Equation (1) and Kontar et al. 2019). At the same time, we investigate different relative density fluctuation levels for fundamental emissions, giving spectrum-averaged mean wavenumbers $C_{q}=1200,2300$, and $4300 R_{\odot}^{-1}$ from Equation (6). The temporal evolution, apparent sizes, and locations of radio sources simulated assuming an anisotropic turbulence with an initially instantaneous pointlike emission are shown in
Figure 3. In the top panels, the thin red line represents the temporal evolution of the observed type III-IIIb radio source, with the gray shaded area indicating its decay phase.

The decay times are $\sim 0.32,0.50$, and 0.72 for the three used values of $C_{q}$, respectively. Higher levels of density fluctuations result in longer decay times and larger FWHM sources sizes. For the case of $C_{q}=2300 R_{\odot}^{-1}$, the source size changes from $\sim 280$ to $\sim 430$ in $0.5 \mathrm{~s}$ during the decay phase (blue dots at the bottom of Figure 3(a)), which agree with the values obtained from the LOFAR observations shown by the red line in Figure 3 (Kontar et al. 2017; Sharykin et al. 2018).

The heliocentric angle $\theta$ affects the apparent centroid position but has almost no influence on the time profiles and source sizes, as seen in Figure 3(b). The centroid shifts (relative to the centroid positions at the peak times) are shown in the middle row, with the red line corresponding to the LOFAR observations.

The effects of the anisotropy parameter $\alpha$ (defined in Equation (3)) on the observed source properties are demonstrated in Figure 3(c) for $\alpha=0.2,0.25$, and 0.3. The decay times are $0.24,0.50$, and $1.01 \mathrm{~s}$, respectively, and the apparent source sizes range from $\sim 270$ to $400 \operatorname{arcmin}^{2}, \sim 280$ to $430 \mathrm{arcmin}^{2}$, and $\sim 280$ to $380 \operatorname{arcmin}^{2}$ during the decay phase, respectively, for the different anisotropy values. The results show that stronger anisotropy yields a shorter time duration and a larger areal expansion rate.

The best fit for the fundamental source observed at $32 \mathrm{MHz}$ assuming instantaneous emission from a point source is provided by the model in which the point source is located at a heliocentric angle $\theta=5^{\circ}$, turbulence with anisotropy $\alpha=0.25$, and $C_{q}=2300 R_{\odot}^{-1}$. The comparison of the observed and simulated lightcurves suggests that the intrinsic duration of fundamental emission cannot be longer than $0.3 \mathrm{~s}$; otherwise, the observed profile will be too long. For Gaussian sources, the observed source area is the sum of the intrinsic area and the area due to scattering. A comparison of the observations to the simulations reveals that intrinsic areas are smaller than $\sim 50 \operatorname{arcmin}^{2}$; larger intrinsic sizes would contradict observations producing the expansion rate below the observed rate.

\subsection{Harmonic Emission}

We also simulate the harmonic component of radio waves emitted at $2.2 R_{\odot}$ (where the local plasma frequency is $16 \mathrm{MHz}$ ) propagating in an anisotropic turbulence medium, and we compare the results with the harmonic source observed by LOFAR at $32.5 \mathrm{MHz}$. Figure 4 shows the simulation results obtained by varying $C_{q}$ (column (a)), the heliocentric angle (column (b)), and the level of anisotropy (column (c)). The parameters used for Figure 4 are the same as the parameters used when simulating the fundamental emission component (see Figure 3).

Simulations with $C_{q}=2300 R_{\odot}, \alpha=0.25, \theta=5^{\circ}$, and an instantaneous point source produce a time profile, source positions, and areas that are similar to the results of the fundamental emission simulations. Due to propagation effects, harmonic emission is found to have a $\sim 0.4$ s decay time, which is much shorter than the $3 \mathrm{~s}$ observed by LOFAR (red thin line in Figure 4). The simulated area of the harmonic emission source is about $\sim 300 \operatorname{arcmin}^{2}$ near the peak, which is comparable to the fundamental emission source area, but smaller than the observed $\sim 500 \operatorname{arcmin}^{2}$. Furthermore, the 

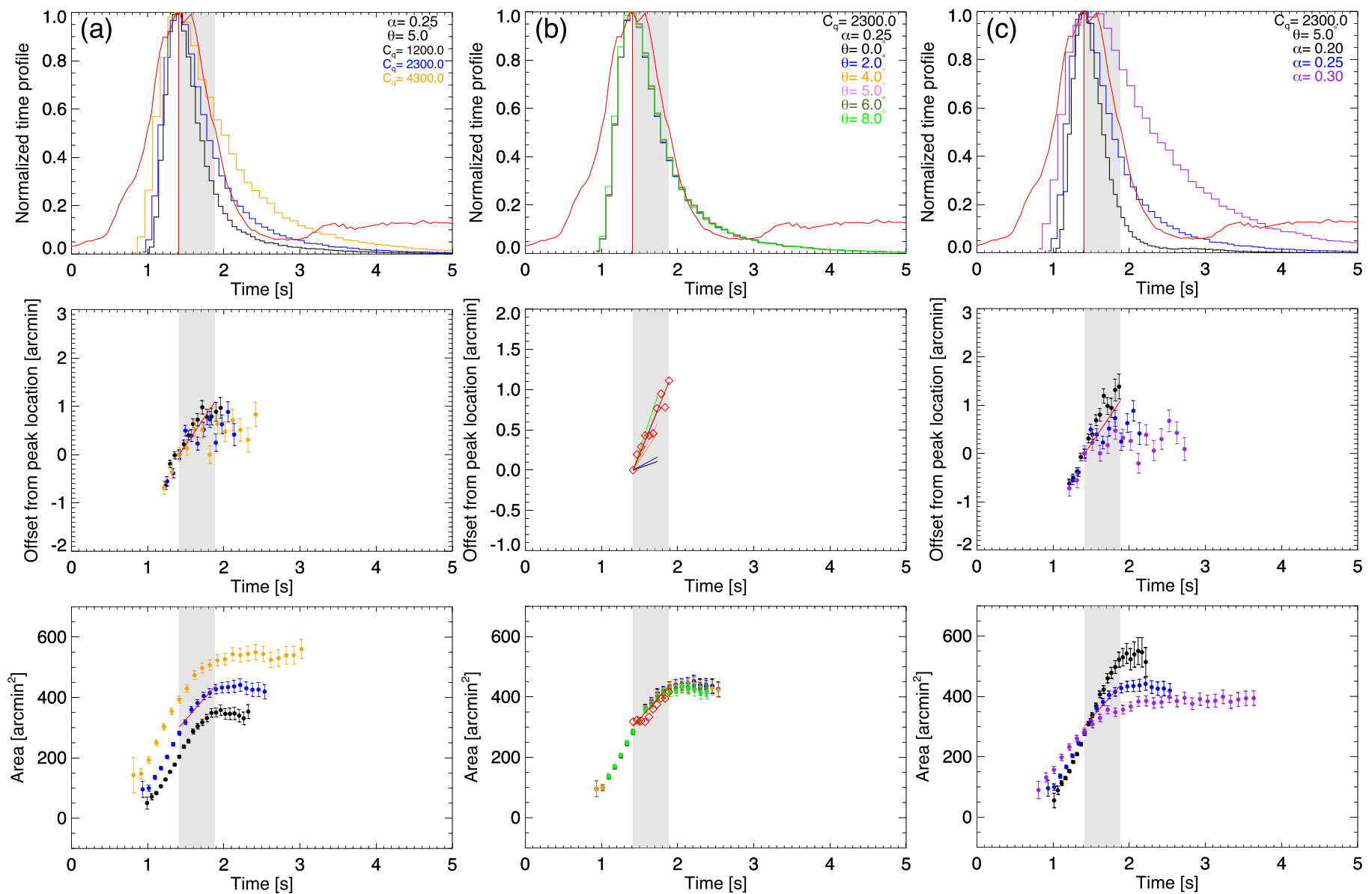

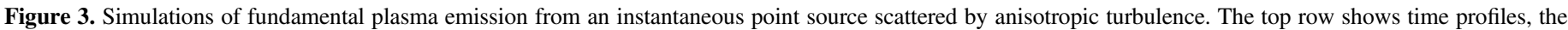

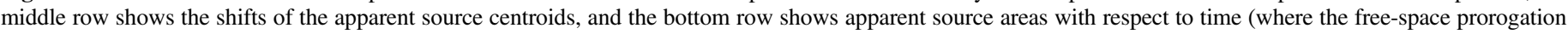

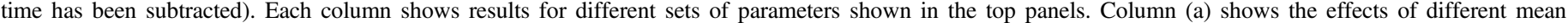

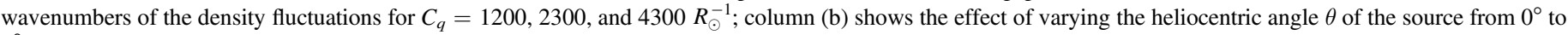

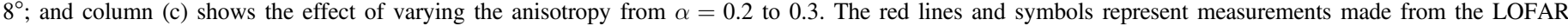

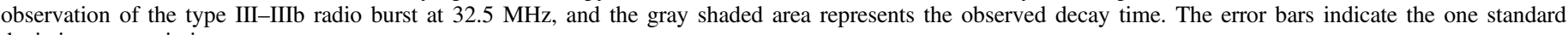
deviation uncertainties.

simulated harmonic source area changes rapidly with time, and the centroids demonstrate rapid motion, similar to that of the fundamental emission sources but inconsistent with the LOFAR observations of the harmonic component.

All of these results (the discrepancy in the time profiles, source motions, and sizes) suggest that the harmonic emission cannot be explained by an instantaneous point source and that it has a finite time duration and size. Indeed, if the fundamental emission is produced at $1.8 R_{\odot}$ and the harmonic at $2.2 R_{\odot}$, i.e., $\Delta r=0.4 R_{\odot}$ away from the fundamental source, the time-offlight spread of electrons could produce a finite harmonic emission duration. According to the observations, the drift rate of the type IIIb burst gives an electron speed of approximately $c / 3$, where $c$ is the speed of light (see Kontar et al. 2017 for details). If the electron beam has a uniform spread of velocities between $c / 6$ and $c / 3$, the time-of-flight duration of electrons at the harmonic location would be $\Delta t=3 \Delta r / c \sim 3 \mathrm{~s}$. Selfconsistent simulations of electron transport (Reid \& Kontar 2018) support such an expansion of the electron beam and an increase of the emission duration with distance. In addition, there is a finite time required for the production of harmonic emission in a given location (e.g., Ratcliffe et al. 2014; Yoon 2018). Therefore, when the estimated electron time-of-flight is taken into account, the duration of harmonic emission could be $3-4 \mathrm{~s}$.

To simulate the effect of finite harmonic emission time, we assume that the harmonic emission is a Gaussian pulse $\exp \left(-t^{2} / 2 \sigma^{2}\right)$ with $\sigma=2 \mathrm{~s}$ (see the caption of Figure 5). Then the observed profile is the convolution of the intrinsic emission and the broadening due to scattering. In addition, we assume that the harmonic source has a finite emission area of 200, 250, and $300 \operatorname{arcmin}^{2}$. The results are shown in Figure 5. Importantly, it can be seen that prolonged emission at the source results in a smaller motion of the centroid compared to the instantaneous injection in Figure 4. Moreover, while the instantaneous harmonic source shows a fast source motion (Figure 4), the $4 \mathrm{~s}$ harmonic emission does not have a clear motion, which is more consistent with the LOFAR observations.

The comparison of the observed harmonic emission with the simulations suggests that an emission source with the physical area of up to $\sim 200 \mathrm{arcmin}^{2}$ will be consistent with the observations. At the same time, in order to explain the slow centroid motion of harmonic emissions and the areal expansion, a continuous harmonic emission lasting $\sim 4 \mathrm{~s}$ is required. 

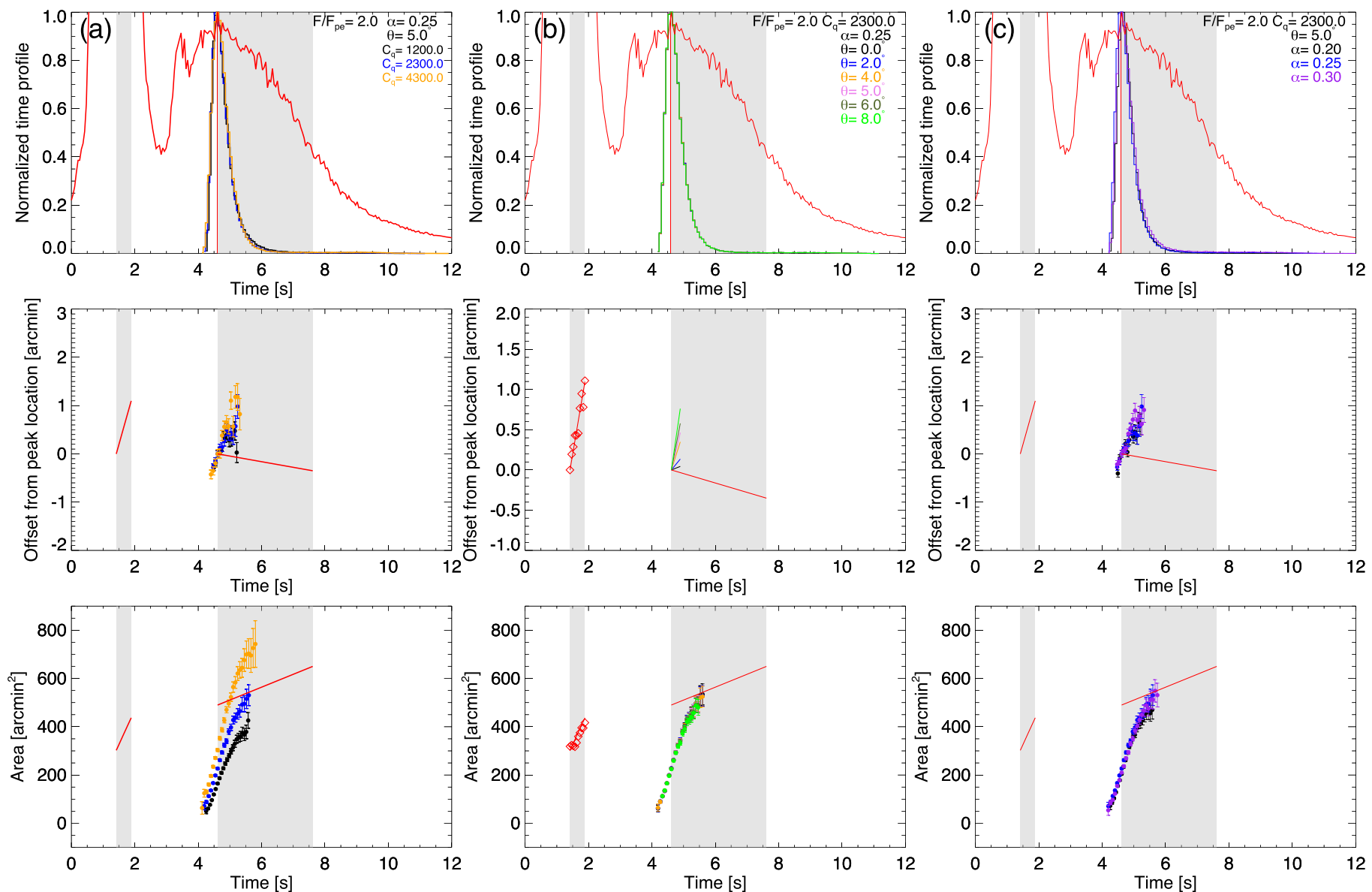

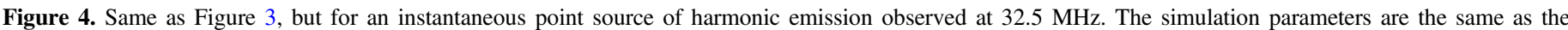
parameters in Figure 3.

\section{Summary}

We quantitatively investigated the way in which scattering of radio waves on random density fluctuations with a power-law spectrum affects the time profile evolution, sizes, and positions of the observed radio bursts emitted via the plasma emission mechanism. Although comparison was made to type III bursts, similar arguments are applicable to all bursts emitted via the plasma emission mechanism, e.g., noise storms (e.g., Mercier et al. 2015), type II solar radio bursts (e.g., Chrysaphi et al. 2020), drifting pairs (e.g., Kuznetsov \& Kontar 2019; Kuznetsov et al. 2020), and probably type IV bursts (e.g., Gordovskyy et al. 2019).

Density fluctuations in the corona lead to angular broadening of the sources, so that the observed source area is increased by scattering. Larger density fluctuations (i.e., larger $C_{q}$ values) would also lead to a longer duration of the wave propagation and increase the duration of the observed emission pulse. As a result, the observed decay times and the observed peak time of the emission increase with higher levels of density fluctuations.

Unlike isotropic turbulence, anisotropic turbulence produces an apparent motion of the source with time, with the apparent velocity depending on the source heliocentric angle (i.e., the angle between the line of sight and the direction from the center of the Sun to the physical source position). Sources with large projection angles (i.e., located close to the limb) experience larger radial displacements, as shown in Figure 3. The time profile remains rather similar for the small heliocentric angles studied. Strong anisotropy ( $\alpha \ll 1$, i.e., density fluctuations being mostly perpendicular) means that the effects occur predominantly along the perpendicular direction to the radial magnetic field. This effect is similar to the observed ellipticity of galactic radio sources observed through the solar corona, where sources are broadened more strongly along the tangential to the solar limb direction (e.g., Hewish 1958; Dennison \& Blesing 1972).

By matching the main characteristics (size, position, and time profile) of the observed sources with those observed by LOFAR, we have estimated the properties of plasma turbulence in the solar corona. The simulations reproduced the subsecond evolution of the source area, position, and time profile of both fundamental and harmonic radio sources. We find that radiowave scattering due to turbulence with spectrum-weighted mean wavenumbers of density fluctuations $C_{q} \sim 2300 R_{\odot}^{-1}$, a source heliocentric angle $\theta=5^{\circ}$, and an anisotropy $\alpha \sim 0.25$ can explain the decay time, apparent source motion, and the temporal evolution of the source size for the fundamental emission of the type III-IIIb radio burst observed by LOFAR. We also simulated the harmonic emission using the same parameters as for the fundamental emission, and found that it can be explained by the same parameters of turbulence when the harmonic has a finite source and finite emission time. The intrinsic source, which is located at a heliocentric angle $\theta=5^{\circ}$, has an area of $200 \operatorname{arcmin}^{2}$ (i.e., FWHM diameter $\sim 16^{\prime}$ ) without scattering and has an FWHM duration of $4.7 \mathrm{~s}$. Then the observed size due to scattering becomes $480 \operatorname{arcmin}^{2}$ with a slightly longer duration of $4.8 \mathrm{~s}$. Another interesting result is 

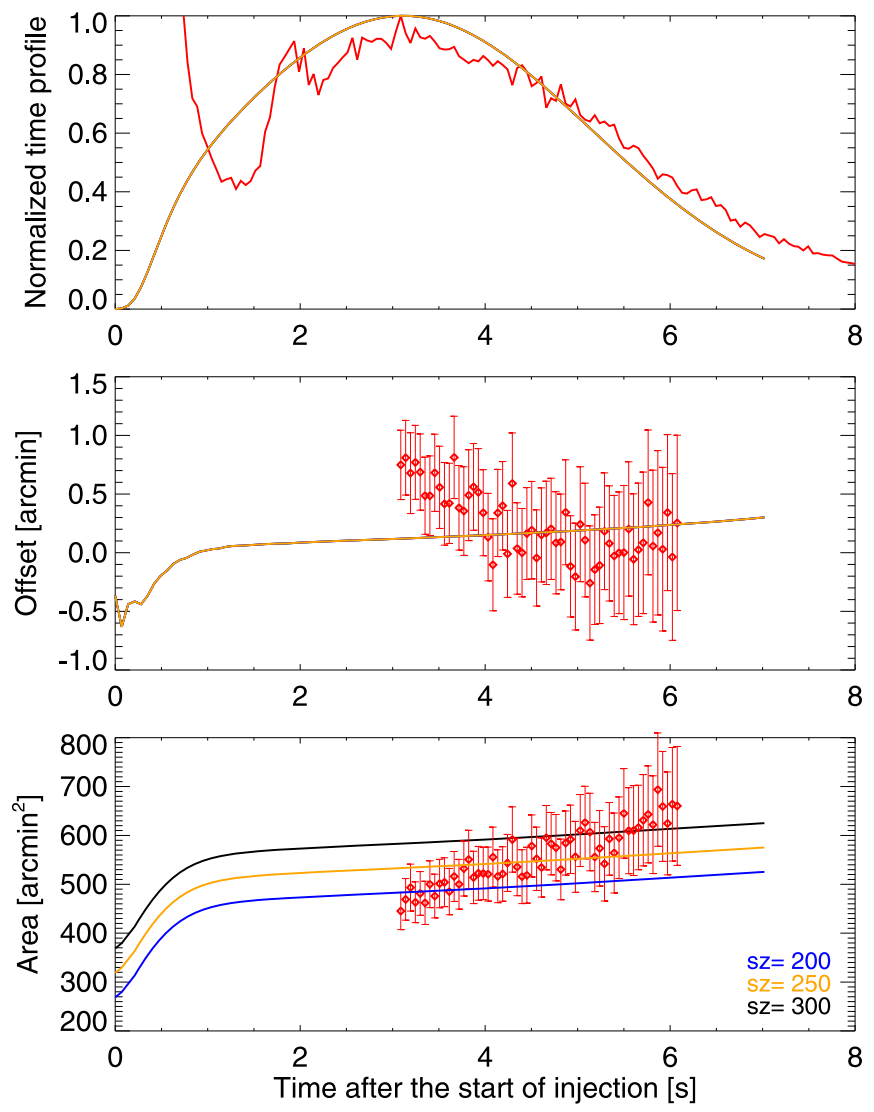

Figure 5. From top to bottom, the solid black lines show the time profile, the centroid position, and area for the harmonic emission obtained assuming a Gaussian profile $\exp \left(-\left(t-t_{0}\right)^{2} /\left(2 \times 2^{2}\right)\right)$ and a finite area of $200,250,300$ $\operatorname{arcmin}^{2}$. All other parameters are the same as those used in Figure 4. The red data represent the observed time profile, centroid position, and size for the harmonic component of the type III-IIIb burst observed with LOFAR, and the associated errors.

that a continuous emission of harmonic radiation for over $4 \mathrm{~s}$ is required to explain the small change in position and size of the harmonic source at $32.5 \mathrm{MHz}$. The intrinsic duration of harmonic emission is likely to be related to electron transport effects. Using the obtained parameters, we produced an image showing the fundamental and harmonic sources (imaged at $32.5 \mathrm{MHz}$ ), presented in Figure 6. It is evident that the simulated sources successfully reproduce the observed sources, shown by Figure 2 in Kontar et al. (2017).

The agreement with the LOFAR observations suggests that $C_{q}=2300 R_{\odot}^{-1}$, so when the density fluctuations are $\epsilon \simeq 0.1$ (e.g., Hahn et al. 2018), the characteristic density scale $h$ should be about $5.5 \times 10^{-5} R_{\odot} \sim 38 \mathrm{~km}$. In the present work, we used an extrapolation of the inner and outer scale measurements from distances $>2 R_{\odot}$, but a more rigorous analysis and multifrequency observations are needed to determine the spatial behavior of $\overline{q \epsilon^{2}}$ throughout the corona and the solar wind.

The results and conclusions of our study can be summarized as follows:

1. Isotropic scattering cannot simultaneously describe all the observed radio emission characteristics, but instead an anisotropic scattering description is required to do so.

2. The apparent source displacement becomes larger for larger heliocentric angles $\theta$, whereas the source area and the time profile are affected to a lesser extent.

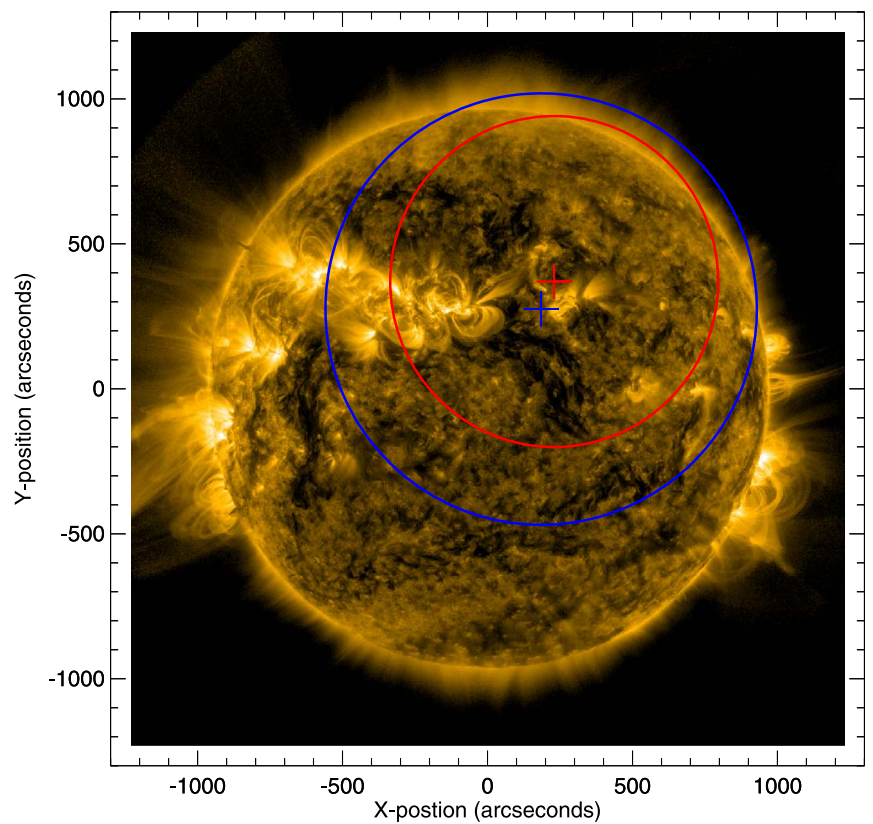

Figure 6. Simulated fundamental (blue contour) and harmonic (red contour) radio sources, overlaid on an SDO/AIA $171 \AA$ image showing the solar surface (at $\sim 11: 57 \mathrm{UT}$ ) in EUV wavelengths. The sources were simulated using $C_{q} \sim 2300 R_{\odot}^{-1}, \theta=5^{\circ}$, and $\alpha \sim 0.25$. The harmonic source was simulated using the injection profile of a finite emission area of $200 \mathrm{arcmin}^{2}$ shown in Figure 5 .

3. The time evolution of the radio flux, source size, and centroid motions of a type III-IIIb burst, for both its fundamental and harmonic components, can be successfully reproduced using the ray-tracing simulations applied.

4. The spectrum-weighted mean wavenumber of the density fluctuations $\left(C_{q}\right)$, the level of anisotropy $(\alpha)$, and the heliocentric angle $(\theta)$ of a type III-IIIb burst are estimated, by comparing its observed characteristics to radio-wave propagation simulations.

The comparison of simulations to observations of source sizes and positions at subsecond timescales allows us to understand the propagation of radio waves in the corona. Radio-wave propagation effects should be taken into account when inferring physical parameters using solar radio-imaging observations, given that the observed radio properties do not represent the intrinsic source properties. The simulations presented are applicable for all types of radio bursts that are generated via the plasma emission mechanism. We note, however, that the density turbulence may not be the same for different events and different frequencies. Therefore, a statistical study of spectral and imaging radio observations that are compared with radio-wave propagation simulations is required in the future.

The work is supported by NSFC grants 11790301, 11973057, 12003048, and the National Key $\mathrm{R} \backslash \& \mathrm{D}$ Program of China 2018 YFA0404602.

\section{ORCID iDs}

Xingyao Chen (ib https://orcid.org/0000-0002-1810-6706

Eduard P. Kontar (i) https:// orcid.org/0000-0002-8078-0902

Nicolina Chrysaphi (iD https://orcid.org/0000-0002-4389-5540 
Natasha L. S. Jeffrey @i https://orcid.org/0000-00016583-1989

Mykola Gordovskyy (1) https://orcid.org/0000-00032291-4922

Baolin Tan (1) https://orcid.org/0000-0003-2047-9664

\section{References}

Arzner, K., \& Magun, A. 1999, A\&A, 351, 1165

Aubier, M., Leblanc, Y., \& Boischot, A. 1971, A\&A, 12, 435

Bastian, T. S. 1994, ApJ, 426, 774

Bian, N. H., Emslie, A. G., \& Kontar, E. P. 2019, ApJ, 873, 33

Bougeret, J. L., \& Steinberg, J. L. 1977, A\&A, 61, 777

Celnikier, L. M., Harvey, C. C., Jegou, R., Moricet, P., \& Kemp, M. 1983, A\&A, 126, 293

Chen, X., Kontar, E. P., Yu, S., et al. 2018, ApJ, 856, 73

Chrysaphi, N., Kontar, E. P., Holman, G. D., \& Temmer, M. 2018, ApJ, 868, 79

Chrysaphi, N., Reid, H. A. S., \& Kontar, E. P. 2020, ApJ, 893, 115

Coles, W. A., \& Harmon, J. K. 1989, ApJ, 337, 1023

Dennison, P. A., \& Blesing, R. G. 1972, PASAu, 2, 86

Dulk, G. A. 1985, ARA\&A, 23, 169

Fokker, A. D. 1965 , BAN, 18, 111

Ginzburg, V. L., \& Zhelezniakov, V. V. 1958, SvA, 2, 653

Goldman, M. V. 1983, SoPh, 89, 403

Gordovskyy, M., Kontar, E., Browning, P., \& Kuznetsov, A. 2019, ApJ, 873,48

Hahn, M., D’Huys, E., \& Savin, D. W. 2018, ApJ, 860, 34

Hewish, A. 1958, MNRAS, 118, 534

Jeffrey, N. L. S., \& Kontar, E. P. 2011, A\&A, 536, A93

Kolotkov, D. Y., Nakariakov, V. M., \& Kontar, E. P. 2018, ApJ, 861, 33
Kontar, E. P., Chen, X., Chrysaphi, N., et al. 2019, ApJ, 884, 122

Kontar, E. P., Yu, S., Kuznetsov, A. A., et al. 2017, NatCo, 8, 1515

Krupar, V., Maksimovic, M., Kontar, E. P., et al. 2018, ApJ, 857, 82

Krupar, V., Szabo, A., Maksimovic, M., et al. 2020, ApJS, 246, 57

Kuznetsov, A. A., Chrysaphi, N., Kontar, E. P., \& Motorina, G. 2020, ApJ, 898, 94

Kuznetsov, A. A., \& Kontar, E. P. 2019, A\&A, 631, L7

Lifshitz, E. M., \& Pitaevskii, L. P. 1981, Physical Kinetics (Oxford: Pergamon)

Manoharan, P. K., Ananthakrishnan, S., \& Pramesh Rao, A. 1987, in Sixth International Solar Wind Conference, ed. V. J. Pizzo, T. Holzer, \& D. G. Sime (Boulder, CO: National Center for Atmospheric Research), 55

Mercier, C., Subramanian, P., Chambe, G., \& Janardhan, P. 2015, A\&A, 576, A136

Parker, E. N. 1960, ApJ, 132, 821

Pécseli, H. 2012, Waves and Oscillations in Plasmas (Boca Raton, FL: CRC Press)

Ramesh, R., Mugundhan, V., \& Prabhu, K. 2020, ApJL, 889, L25

Ratcliffe, H., Kontar, E. P., \& Reid, H. A. S. 2014, A\&A, 572, A111

Reid, H. A. S., \& Kontar, E. P. 2018, A\&A, 614, A69

Riddle, A. C. 1974, SoPh, 35, 153

Robinson, R. D. 1983, PASAu, 5, 208

Shaikh, D., \& Zank, G. P. 2010, MNRAS, 402, 362

Sharykin, I. N., Kontar, E. P., \& Kuznetsov, A. A. 2018, SoPh, 293, 115

Steinberg, J. L. 1972, A\&A, 18, 382

Steinberg, J. L., Aubier-Giraud, M., Leblanc, Y., \& Boischot, A. 1971, A\&A, 10,362

Thejappa, G., MacDowall, R. J., \& Kaiser, M. L. 2007, ApJ, 671, 894

Tu, C.-Y., \& Marsch, E. 1993, JGR, 98, 1257

van Haarlem, M. P., Wise, M. W., Gunst, A. W., et al. 2013, A\&A, 556, A2

Wohlmuth, R., Plettemeier, D., Edenhofer, P., et al. 2001, SSRv, 97, 9

Yoon, P. H. 2018, PhPl, 25, 011603

Yoon, P. H., Ziebell, L. F., Kontar, E. P., \& Schlickeiser, R. 2016, PhRvE, 93, 033203 